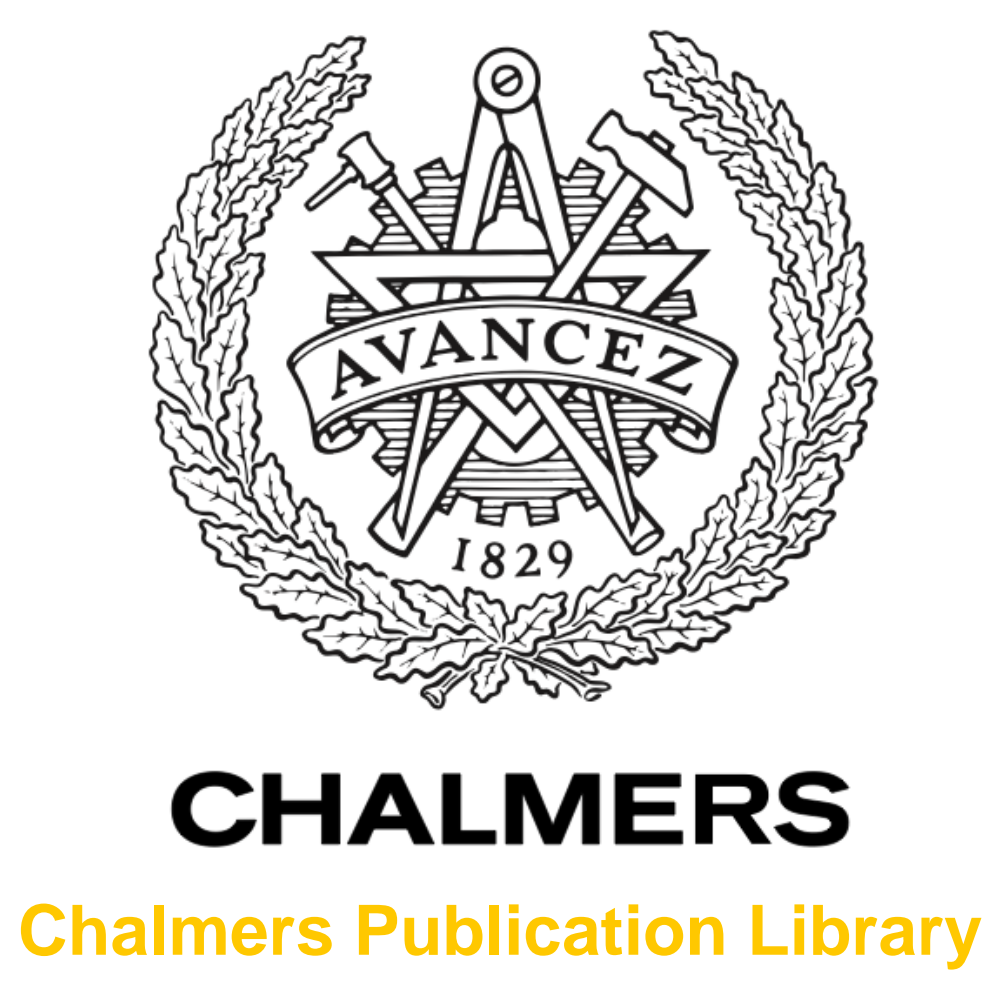

Performance analysis of large scale MU-MIMO with optimal linear receivers

This document has been downloaded from Chalmers Publication Library (CPL). It is the author's version of a work that was accepted for publication in:

2012 Swedish Communication Technologies Workshop, Swe-CTW 2012, Lund, 24 - 26

\title{
October 2012
}

Citation for the published paper:

Ngo, H. ; Matthaiou, M. ; Larsson, E. (2012) "Performance analysis of large scale MUMIMO with optimal linear receivers". 2012 Swedish Communication Technologies

Workshop, Swe-CTW 2012, Lund, 24 - 26 October 2012 pp. 59-64.

http://dx.doi.org/10.1109/Swe-CTW.2012.6376290

Downloaded from: http://publications.lib.chalmers.se/publication/170610

Notice: Changes introduced as a result of publishing processes such as copy-editing and formatting may not be reflected in this document. For a definitive version of this work, please refer to the published source. Please note that access to the published version might require a subscription.

Chalmers Publication Library (CPL) offers the possibility of retrieving research publications produced at Chalmers University of Technology. It covers all types of publications: articles, dissertations, licentiate theses, masters theses, conference papers, reports etc. Since 2006 it is the official tool for Chalmers official publication statistics. To ensure that Chalmers research results are disseminated as widely as possible, an Open Access Policy has been adopted.

The CPL service is administrated and maintained by Chalmers Library. 


\title{
Performance Analysis of Large Scale MU-MIMO with Optimal Linear Receivers
}

\author{
Hien Quoc Ngo*, Michail Matthaiou ${ }^{\dagger}$, and Erik G. Larsson* \\ * Department of Electrical Engineering (ISY), Linköping University, Linköping, Sweden, emails:\{nqhien, egl\}@isy.liu.se \\ $\dagger^{\dagger}$ Department of Signals and Systems, Chalmers University of Technology, Gothenburg, Sweden, email: michail.matthaiou@chalmers.se
}

\begin{abstract}
We consider the uplink of multicell multiuser MIMO (MU-MIMO) systems with very large antenna arrays at the base station (BS). We assume that the BS estimates the channel through uplink training, and then uses this channel estimate to detect the signals transmitted from a multiplicity of autonomous users in its cell. By taking the correlation between the channel estimate and the interference from other cells into account, we propose an optimal linear receiver (OLR) which maximizes the received signal-to-interference-plus-noise (SINR). Analytical approximations of the exact and lower bound on the achievable rate are then derived. The bound is very tight, especially at large number of BS antennas. We show that at low SINR, maximalratio combing (MRC) receiver performs as well as OLR, however at high SINR, OLR outperforms MRC. Compared with the typical minimum mean-square error receiver, our proposed OLR improves systematically the system performance, especially when the interference is large.
\end{abstract}

\section{INTRODUCTION}

MU-MIMO with very large antenna arrays at the BS represents an emerging hot area since it i) offers more degrees of freedom; and ii) offers increased power efficiency [1]-[3]. For such very large systems, the BS can serve a multiplicity of autonomous users in the same time-frequency resource, with each user getting high throughput. Since the number of BS antennas is large, the signal processing at the BS should be simple, e.g., using linear precoders for the downlink and linear detectors for the uplink. Among typical linear detectors at the $\mathrm{BS}, \mathrm{MRC}$ is advantageous since it can be implemented in a distributed manner. From the system performance point of view, in [4], we showed that MRC performs as well as ZF and MMSE in the regime where each user has a throughput of an order of about 1 bit per channel use, whilst MMSE is the optimal linear detector for single-cell systems. However, for multicell MU-MIMO systems, where the channel is estimated at the BS by using uplink pilots, it is unknown whether the typical MMSE receiver is optimal. In this paper, we shed some light onto this very interesting problem. Intuitively, the channel estimate is contaminated by the pilots transmitted from other cells. As a consequence, it correlates with the interference from other cells. Typical MMSE receivers do not take this correlation into account and hence, MMSE receiver is not optimal for these systems. In this paper, we propose a novel OLR which takes the correlation between the channel estimate and the intercell interference into account. The paper makes the following specific contributions:

- We propose an OLR which exploits the correlation between the channel estimates and the interference from other cells, due to the pilot contamination effect. Then we compare the performance between MRC, ZF, MMSE, and OLR receivers.

- We derive new approximating expressions for the statistical distributions of the received SINR. We then deduce closed-form approximations for the exact uplink achievable ergodic rate as well as a tight lower bound on this rate. This analysis provides a benchmark for all conventional linear receivers.

- Finally, we consider the asymptotic performance of OLR when the BS uses a large antenna array. Similar to other linear receivers, for OLR, the transmit power of each user can be made inversely proportional to the square-root of the number of antennas with no performance degradation.

\section{Multicell MU-MiMO System Model}

Consider the uplink transmission of a multicell MU-MIMO system with $L$ cells. Each cell includes one BS equipped with $N$ antennas, and $K$ single-antenna users. We assume that $L$ BSs share the same frequency band. The $N \times 1$ received vector at the $l$ th BS is given by

$$
\boldsymbol{y}_{l}=\sqrt{p_{\mathrm{u}}} \sum_{i=1}^{L} \boldsymbol{G}_{l i} \boldsymbol{x}_{i}+\boldsymbol{n}_{l}
$$

where $\boldsymbol{G}_{l i} \in \mathbb{C}^{N \times K}$ is the channel matrix between the $l$ th BS and $K$ users in the $i$ th cell; $\sqrt{p_{\mathrm{u}}} \boldsymbol{x}_{i} \in \mathbb{C}^{K \times 1}$ is transmitted vector of $K$ users in the $i$ th cell (the average power transmitted by each user is $\left.p_{\mathrm{u}}\right)$; and $\boldsymbol{n}_{l} \in \mathbb{C}^{N \times 1}$ is the AWGN vector, distributed as $\boldsymbol{n}_{l} \sim \mathcal{C N}\left(\mathbf{0}, \boldsymbol{I}_{N}\right)$. Here we assume equal power allocation for all users. Note that this assumption does not affect the obtained results, and can provide a lower bound on the performance of practical systems, where power control is being implemented.

The channel matrix, $\boldsymbol{G}_{l i}$, models independent fast fading, path-loss attenuation, and log-normal shadow fading. ${ }^{1}$ Its elements $g_{\text {limk }}$ are given by

$$
g_{\text {limk }}=h_{\text {limk }} \sqrt{\beta_{l i k}}, \quad m=1,2, \ldots, N
$$

where $h_{\text {limk }} \sim \mathcal{C N}(0,1)$ is the fast Rayleigh fading coefficient from the $k$ th user in the $i$ th cell to the $m$ th antenna of the $l$ th BS, while $\sqrt{ }$ 


\section{A. Channel Estimation}

To detect the signals transmitted from $K$ users in its cell, the BS has to have knowledge of channel state information (CSI). This CSI can be estimated at the BS. In practice, a standard way of estimating the channels is using uplink pilots. During a part of the coherence interval of the channel, all users simultaneously transmit pilot sequences of length $\tau$ symbols ( $\tau$ is smaller than the coherence time of the channel). In each cell, each user is assigned an orthogonal pilot sequence. We assume that the same set of pilot sequences is used in all cells. This assumption comes from the fact that, in practical cellular networks, the channel coherence time typically is not long enough to allow for orthogonality between the pilots in different cells. The MMSE channel estimates for the $k$-th column of the channel matrix $\boldsymbol{G}_{l i}$ is given by [5]

$$
\hat{\boldsymbol{g}}_{l i k}=\beta_{l i k}\left(\sum_{j=1}^{L} \beta_{l j k}+\frac{1}{\tau p_{\mathrm{p}}}\right)^{-1}\left(\sum_{j=1}^{L} \boldsymbol{g}_{l j k}+\frac{1}{\sqrt{\tau p_{\mathrm{p}}}} \boldsymbol{w}_{l k}\right)
$$

where $\boldsymbol{w}_{l k} \sim \mathcal{C N}\left(\mathbf{0}, \boldsymbol{I}_{N}\right)$, and $p_{\mathrm{p}}$ is the transmit power of each pilot symbol. From (3), we see that $\hat{\boldsymbol{g}}_{l i k}=\frac{\beta_{l i k}}{\beta_{l l k}} \hat{\boldsymbol{g}}_{l l k}$, or

$$
\hat{\boldsymbol{G}}_{l i}=\hat{\boldsymbol{G}}_{l l} \boldsymbol{D}_{i}
$$

where $\boldsymbol{D}_{i}=\operatorname{diag}\left\{\frac{\beta_{l i 1}}{\beta_{l l 1}}, \frac{\beta_{l i 2}}{\beta_{l l 2}}, \ldots, \frac{\beta_{l i K}}{\beta_{l l K}}\right\}$, and $\hat{\boldsymbol{G}}_{l i} \triangleq$ $\left[\begin{array}{lll}\hat{\boldsymbol{g}}_{l i 1} & \ldots & \hat{\boldsymbol{g}}_{l i K}\end{array}\right]$. We can now decompose $\boldsymbol{g}_{l i k}$ as

$$
\boldsymbol{g}_{l i k}=\hat{\boldsymbol{g}}_{l i k}+\boldsymbol{\varepsilon}_{l i k}
$$

where $\varepsilon_{l l k}$ is the estimation error. We have

$$
\hat{\boldsymbol{g}}_{l i k} \sim \mathcal{C N}\left(\mathbf{0}, \frac{\beta_{l i k}^{2}}{\sum_{j=1}^{L} \beta_{l j k}+1 /\left(\tau p_{\mathrm{p}}\right)} \boldsymbol{I}_{M}\right)
$$

and

$$
\boldsymbol{\varepsilon}_{l i k} \sim \mathcal{C N}\left(\mathbf{0}, \beta_{l i k}\left(1-\frac{\beta_{l i k}}{\sum_{j=1}^{L} \beta_{l j k}+1 /\left(\tau p_{\mathrm{p}}\right)}\right) \boldsymbol{I}_{M}\right) .
$$

Since we use MMSE channel estimation, the channel estimate $\hat{\boldsymbol{g}}_{l i k}$ is independent of the estimation error $\boldsymbol{\varepsilon}_{l i k}$ [6].

\section{B. Linear Receivers}

We consider linear detection schemes at the BS. Let $\boldsymbol{A}_{l}$ be an $N \times K$ linear detection matrix which depends on the channel estimates $\hat{\boldsymbol{G}}_{l i}, i=1, \ldots, L$. From (4), $\boldsymbol{A}_{l}$ can be represented as a function of $\hat{\boldsymbol{G}}_{l l}$. The $l$ th BS processes its received signal by multiplying it by $\boldsymbol{A}_{l}^{H}$ as follows

$$
\boldsymbol{r}_{l}=\boldsymbol{A}_{l}^{H} \boldsymbol{y}_{l}=\sqrt{p_{\mathrm{u}}} \boldsymbol{A}_{l}^{H} \sum_{i=1}^{L} \boldsymbol{G}_{l i} \boldsymbol{x}_{i}+\boldsymbol{A}_{l}^{H} \boldsymbol{n}_{l} .
$$

Let $\boldsymbol{a}_{l k}$ be the $k$ th column of $\boldsymbol{A}_{l}$. Then the $k$ th element of $\boldsymbol{r}_{l}$ is

$$
\begin{aligned}
r_{l k} & =\sqrt{p_{\mathrm{u}}} \boldsymbol{a}_{l k}^{H} \hat{\boldsymbol{g}}_{l l k} x_{l k}+\sqrt{p_{\mathrm{u}}} \sum_{j \neq k}^{K} \boldsymbol{a}_{l k}^{H} \hat{\boldsymbol{g}}_{l l j} x_{l j}+\sqrt{p_{\mathrm{u}}} \boldsymbol{a}_{l k}^{H} \boldsymbol{\mathcal { E }}_{l l} \boldsymbol{x}_{l} \\
& +\sqrt{p_{\mathrm{u}}} \boldsymbol{a}_{l k}^{H} \sum_{i \neq l}^{L} \hat{\boldsymbol{G}}_{l i} \boldsymbol{x}_{i}+\sqrt{p_{\mathrm{u}}} \boldsymbol{a}_{l k}^{H} \sum_{i \neq l}^{L} \mathcal{E}_{l i} \boldsymbol{x}_{i}+\boldsymbol{a}_{l k}^{H} \boldsymbol{n}_{l}
\end{aligned}
$$

where $x_{l k}$ is the $k$ th element of $\boldsymbol{x}_{l}$ and $\mathcal{E}_{l i} \triangleq\left[\begin{array}{llll}\varepsilon_{l i 1} & \ldots & \varepsilon_{l i K}\end{array}\right]$. Since $\hat{\boldsymbol{G}}_{l i}$ and $\mathcal{E}_{l i}$ are independent, the SINR of the uplink transmission from the $k$ th user in $l$ th cell to its BS is given by

$$
\begin{aligned}
& \operatorname{SINR}_{k}= \\
& \frac{p_{\mathrm{u}}\left|\boldsymbol{a}_{l k}^{H} \hat{\boldsymbol{g}}_{l l k}\right|^{2}}{p_{\mathrm{u}} \sum_{j \neq k}^{K}\left|\boldsymbol{a}_{l k}^{H} \hat{\boldsymbol{g}}_{l l j}\right|^{2}+p_{\mathrm{u}} \boldsymbol{a}_{l k}^{H} \boldsymbol{R}_{\varepsilon} \boldsymbol{a}_{l k}+p_{\mathrm{u}} \sum_{i \neq l}^{L}\left\|\boldsymbol{a}_{l k}^{H} \hat{\boldsymbol{G}}_{l i}\right\|^{2}+\left\|\boldsymbol{a}_{l k}\right\|^{2}}
\end{aligned}
$$

where

$$
\boldsymbol{R}_{\varepsilon} \triangleq \sum_{i=1}^{L} \mathbb{E}\left\{\mathcal{E}_{l i} \mathcal{E}_{l i}^{H}\right\}=\sum_{i=1}^{L} \gamma_{l i} \boldsymbol{I}_{M}
$$

and where

$$
\gamma_{l i}=\sum_{k=1}^{K}\left[\beta_{l i k}-\beta_{l i k}^{2}\left(\sum_{j=1}^{L} \beta_{l j k}+\frac{1}{\tau p_{\mathrm{p}}}\right)^{-1}\right] .
$$

Note that the above SINR can be approached if the message is encoded over many realizations of all sources of randomness in the model (noise and channel estimate error) [4].

\section{OPTIMAL LiNEAR RECEIVERS}

In this section, we derive a novel OLR which maximizes the received SINR given by (7). We then pursue a statistical characterization of the corresponding optimal SINR. Substituting (8) into (7), and after some algebraic manipulations, we obtain

$$
\operatorname{SINR}_{k}=\frac{\left|\boldsymbol{a}_{l k}^{H} \hat{\boldsymbol{g}}_{l l k}\right|^{2}}{\boldsymbol{a}_{l k}^{H} \boldsymbol{\Xi}_{k} \boldsymbol{a}_{l k}}
$$

where $\boldsymbol{\Xi}_{k} \triangleq \sum_{j \neq k}^{K} \hat{\boldsymbol{g}}_{l l j} \hat{\boldsymbol{g}}_{l l j}^{H}+\sum_{i \neq l}^{L} \hat{\boldsymbol{G}}_{l i} \hat{\boldsymbol{G}}_{l i}^{H}+\left(\sum_{i=1}^{L} \gamma_{l i}+\frac{1}{p_{\mathrm{u}}}\right) \boldsymbol{I}_{M}$. Using Cauchy-Schwarz's inequality, we have

$$
\begin{aligned}
\operatorname{SINR}_{k} & =\frac{\left|\boldsymbol{a}_{l k}^{H} \hat{\boldsymbol{g}}_{l l k}\right|^{2}}{\boldsymbol{a}_{l k}^{H} \boldsymbol{\Xi}_{k} \boldsymbol{a}_{l k}}=\frac{\left|\boldsymbol{a}_{l k}^{H} \boldsymbol{\Xi}_{k}^{1 / 2} \boldsymbol{\Xi}_{k}^{-1 / 2} \hat{\boldsymbol{g}}_{l l k}\right|^{2}}{\boldsymbol{a}_{l k}^{H} \boldsymbol{\Xi}_{k} \boldsymbol{a}_{l k}} \\
& \leq \frac{\left\|\boldsymbol{a}_{l k}^{H} \boldsymbol{\Xi}_{k}^{1 / 2}\right\|^{2}\left\|\boldsymbol{\Xi}_{k}^{-1 / 2} \hat{\boldsymbol{g}}_{l l k}\right\|^{2}}{\boldsymbol{a}_{l k}^{H} \boldsymbol{\Xi}_{k} \boldsymbol{a}_{l k}}=\left\|\boldsymbol{\Xi}_{k}^{-1 / 2} \hat{\boldsymbol{g}}_{l l k}\right\|^{2} .
\end{aligned}
$$

The equality holds when $\boldsymbol{a}_{l k}=c \boldsymbol{\Xi}_{k}^{-1} \hat{\boldsymbol{g}}_{l l k}$, for any $c \in \mathbb{C}$, $c \neq 0$. Note that the choice of $c$ does not affect the system performance. Therefore the optimal linear detector at the $l$ th $\mathrm{BS}$ is given by

$$
\boldsymbol{a}_{l k}=c \boldsymbol{\Xi}_{k}^{-1} \hat{\boldsymbol{g}}_{l l k} .
$$

Remark 1: Note that in typical MMSE receivers, the intercell interference is treated as uncorrelated noise, such that

$\boldsymbol{a}_{l k}=\left(\hat{\boldsymbol{G}}_{l l} \hat{\boldsymbol{G}}_{l l}^{H}+\mathbb{E}\left\{\boldsymbol{\mathcal { E }}_{l l} \boldsymbol{\mathcal { E }}_{l l}^{H}\right\}+\sum_{i \neq l}^{L} \mathbb{E}\left\{\boldsymbol{G}_{l i} \boldsymbol{G}_{l i}^{H}\right\}+\frac{1}{p_{\mathrm{u}}} \boldsymbol{I}_{M}\right)^{-1} \hat{\boldsymbol{g}}_{l l k}$.

By contrast, our OLR takes the correlation between the channel estimate and the intercell interference into account. 
This improves the system performance, especially in strong intercell interference conditions.

Remark 2: From (12) and (13), we can see that when the intercell interference is small, the typical MMSE approaches the OLR. Furthermore, it is well known that the performances of MRC and ZF approach the performance of MMSE at low and high SNRs, respectively. Therefore, the performance analysis of our proposed OLR is generic, i.e., it can be used to analyze the performance of systems with linear receivers such as MRC, ZF, and MMSE. Note that, to the best of the authors' knowledge, even with MMSE receivers, there is no analysis of the considered multicell MU-MIMO system for finite $N$.

From (11), with OLR, the SINR of the uplink transmission from the $k$ th user is given by

$$
\operatorname{SINR}_{\mathrm{opt}, k}=\left\|\Xi_{k}^{-1 / 2} \hat{\boldsymbol{g}}_{l l k}\right\|^{2} .
$$

Using a similar methodology as in [7], we obtain

$$
\operatorname{SINR}_{\mathrm{opt}, k}=\frac{1}{1-\rho_{k}}-1
$$

where

$$
\rho_{k} \triangleq \hat{\boldsymbol{g}}_{l l k}^{H}\left(\sum_{i=1}^{L} \hat{\boldsymbol{G}}_{l i} \hat{\boldsymbol{G}}_{l i}^{H}+\left(\sum_{i=1}^{L} \gamma_{l i}+\frac{1}{p_{\mathrm{u}}}\right) \boldsymbol{I}_{N}\right)^{-1} \hat{\boldsymbol{g}}_{l l k} .
$$

From (4), we have

$$
\rho_{k}=\hat{\boldsymbol{g}}_{l l k}^{H}\left(\hat{\boldsymbol{G}}_{l l} \boldsymbol{D} \hat{\boldsymbol{G}}_{l l}^{H}+\left(\sum_{i=1}^{L} \gamma_{l i}+\frac{1}{p_{\mathrm{u}}}\right) \boldsymbol{I}_{N}\right)^{-1} \hat{\boldsymbol{g}}_{l l k}
$$

where $\boldsymbol{D} \triangleq \sum_{i=1}^{L} D_{i}^{2}$. Using the matrix inversion lemma, and the property $(\boldsymbol{A B})^{-1}=\boldsymbol{B}^{-1} \boldsymbol{A}^{-1}$, where $\boldsymbol{A}$ and $\boldsymbol{B}$ are invertible square matrices, we obtain

$$
\begin{aligned}
\rho_{k} & =\left[\hat{\boldsymbol{G}}_{l l}^{H}\left(\hat{\boldsymbol{G}}_{l l} \boldsymbol{D} \hat{\boldsymbol{G}}_{l l}^{H}+\left(\sum_{i=1}^{L} \gamma_{l i}+\frac{1}{p_{\mathrm{u}}}\right) \boldsymbol{I}_{N}\right)^{-1} \hat{\boldsymbol{G}}_{l l}\right]_{k k} \\
& =\left[\left(\left(\frac{\hat{\boldsymbol{G}}_{l l}^{H} \hat{\boldsymbol{G}}_{l l}}{\sum_{i=1}^{L} \gamma_{l i}+\frac{1}{p_{\mathrm{u}}}}\right)^{-1}+\boldsymbol{D}\right)_{k k}^{-1}\right]_{k k} \\
& =\left[\left(\frac{\boldsymbol{D} \hat{\boldsymbol{G}}_{l l}^{H} \hat{\boldsymbol{G}}_{l l} \boldsymbol{D}}{\sum_{i=1}^{L} \gamma_{l i}+\frac{1}{p_{\mathrm{u}}}}+\boldsymbol{D}\right)^{-1}\right]_{k k}
\end{aligned}
$$

Substituting (17) into (15), we obtain

$$
\begin{aligned}
\operatorname{SINR}_{\mathrm{Opt}, k} & =\frac{1}{1-\left[\boldsymbol{D}^{-1}\right]_{k k}+\left[\left(\frac{\boldsymbol{D} \hat{\boldsymbol{G}}_{l l}^{H} \hat{\boldsymbol{G}}_{l l} \boldsymbol{D}}{\sum_{i=1}^{L} \gamma_{l i}+\frac{1}{p_{\mathrm{u}}}}+\boldsymbol{D}\right)^{-1}\right]_{k k}}-1 \\
& =\frac{\left[\boldsymbol{D}^{-1}\right]_{k k} X}{\left(1-\left[\boldsymbol{D}^{-1}\right]_{k k}\right) X+1}
\end{aligned}
$$

where

$$
X \triangleq \frac{1}{\left[\left(\frac{\boldsymbol{D}^{1 / 2} \hat{\boldsymbol{G}}_{l l}^{H} \hat{\boldsymbol{G}}_{l l} \boldsymbol{D}^{1 / 2}}{\sum_{i=1}^{L} \gamma_{l i}+\frac{1}{p_{\mathrm{u}}}}+\boldsymbol{I}_{K}\right)^{-1}\right]_{k k}}-1
$$

The probability density function (PDF) of $X$ can be approximated by a Gamma distribution as follows [8]

$$
p_{X}(x) \approx \frac{x^{\alpha_{k}-1} e^{-x / \theta_{k}}}{\Gamma\left(\alpha_{k}\right) \theta_{k}^{\alpha_{k}}}
$$

where $\Gamma(\cdot)$ is the Gamma function [9, Eq. 8.310.1], while

$$
\begin{aligned}
\alpha_{k} & =\frac{(N-K+1+(K-1) \mu)^{2}}{N-K+1+(K-1) \kappa} \\
\theta_{k} & =\frac{N-K+1+(K-1) \kappa}{N-K+1+(K-1) \mu} \frac{\left(\sum_{i=1}^{L} \gamma_{l i}+\frac{1}{p_{\mathrm{u}}}\right)^{-1} \sum_{i=1}^{L} \beta_{l i k}^{2}}{\left(\sum_{i=1}^{L} \beta_{l i k}+\frac{1}{\tau p_{\mathrm{p}}}\right)}
\end{aligned}
$$

and where $\mu$ and $\kappa$ are determined by solving the following equations:

$$
\begin{array}{r}
\mu=\frac{1}{K-1} \sum_{i=1, i \neq k}^{K} \frac{1}{\tau_{i}\left(1-\frac{K-1}{N}+\frac{K-1}{N} \mu\right)+1} \\
\kappa\left(K-1+\sum_{i=1, i \neq k}^{K} \frac{\tau_{i} \frac{K-1}{N}}{\left(\tau_{i}\left(1-\frac{K-1}{N}+\frac{K-1}{N} \mu\right)+1\right)^{2}}\right) \\
=\sum_{i=1, i \neq k}^{K} \frac{\tau_{i} \mu \frac{K-1}{N}+1}{\left(\tau_{i}\left(1-\frac{K-1}{N}+\frac{K-1}{N} \mu\right)+1\right)^{2}}
\end{array}
$$

where $\tau_{i} \triangleq \frac{N \sum_{j=1}^{L} \beta_{l j i}^{2}}{\left(\sum_{j=1}^{L} \beta_{l j i}+\frac{1}{\tau p_{\mathrm{p}}}\right)\left(\sum_{j=1}^{L} \gamma_{l j}+\frac{1}{p_{\mathrm{u}}}\right)}$. By using the approximate distribution of $X$, we can obtain the approximate distribution of $\mathrm{SINR}_{\mathrm{opt}, k}$ as follows.

Proposition 1: The approximate cumulative distribution function (CDF) and PDF of $\operatorname{SINR}_{\mathrm{Opt}, k}$ are respectively given by (23) and (24) shown at the bottom of the page, where $\gamma(a, x)$ is the incomplete gamma function [9, Eq. (8.350.1)].

$$
\begin{aligned}
& F_{\mathrm{SINR}_{\mathrm{opt}, k}}(z)=\left\{\begin{array}{l}
\frac{1}{\Gamma\left(\alpha_{k}\right)} \gamma\left(\alpha_{k}, \frac{\theta_{k}^{-1}[\boldsymbol{D}]_{k k} z}{1-\left([\boldsymbol{D}]_{k k}-1\right) z}\right), \text { if } z<\frac{1}{[\boldsymbol{D}]_{k k}-1} \\
1, \text { otherwise }
\end{array}\right. \\
& p_{\mathrm{SINR}_{\mathrm{opt}, k}}(z)=\left\{\begin{array}{l}
\frac{[\boldsymbol{D}]_{k k}^{\alpha} z^{\alpha_{k}-1}}{\Gamma\left(\alpha_{k}\right) \theta_{k}^{\alpha}} \frac{1}{\left[1-\left([\boldsymbol{D}]_{k k}-1\right) z\right]^{\alpha k^{+1}}} \exp \left(-\frac{\theta_{k}^{-1}[\boldsymbol{D}]_{k k} z}{1-\left([\boldsymbol{D}]_{k k}-1\right) z}\right), \text { if } z<\frac{1}{[\boldsymbol{D}]_{k k}-1} \\
0, \text { otherwise }
\end{array}\right.
\end{aligned}
$$


Proof: From (18), we have

$$
\begin{aligned}
F_{\text {SINR }_{\mathrm{opt}, k}}(z) & \operatorname{Pr}\left(\frac{\left[\boldsymbol{D}^{-1}\right]_{k k} X}{\left(1-\left[\boldsymbol{D}^{-1}\right]_{k k}\right) X+1} \leq z\right) \\
= & \operatorname{Pr}\left(\left(\left[\boldsymbol{D}^{-1}\right]_{k k}-\left(1-\left[\boldsymbol{D}^{-1}\right]_{k k}\right) z\right) X \leq z\right) \\
= & \left\{\begin{array}{l}
\operatorname{Pr}\left(X \leq \frac{[\boldsymbol{D}]_{k k} z}{1-\left([\boldsymbol{D}]_{k k}-1\right) z}\right), \text { if } z<\frac{1}{[\boldsymbol{D}]_{k k}-1} \\
1, \text { otherwise }
\end{array}\right. \\
= & \left\{\begin{array}{l}
F_{X}\left(\frac{[\boldsymbol{D}]_{k k} z}{1-\left([\boldsymbol{D}]_{k k}-1\right) z}\right), \text { if } z<\frac{1}{[\boldsymbol{D}]_{k k}-1} \\
1, \text { otherwise }
\end{array}\right.
\end{aligned}
$$

where $F_{X}(x)$ is the CDF of $X$ which is given by

$$
F_{X}(x) \approx \int_{0}^{x} \frac{y^{\alpha_{k}-1} e^{-y / \theta_{k}}}{\Gamma\left(\alpha_{k}\right) \theta_{k}^{\alpha_{k}}} d y=\frac{1}{\Gamma\left(\alpha_{k}\right)} \gamma\left(\alpha_{k}, \frac{x}{\theta_{k}}\right)
$$

where the last equation is obtained by using [9, Eq. (3.381.1)]. Substituting (26) into (25), we arrive at the desired result (23).

The PDF of SINR opt,$k$ in (24) follows immediately from differentiating (23) with respect to $z$ using the identity $\frac{d \gamma(a, x)}{d x}=$ $x^{a-1} e^{-x}$ [9, Eq. (8.356.4)].

The distribution of $\mathrm{SINR}_{\mathrm{opt}, k}$ enables us to further evaluate the system performance. For example, the above CDF expression can be used to study the outage probability of the system, i.e., the probability that the instantaneous SINR, SINR opt,$k_{\text {, }}$ falls below a given threshold $\gamma_{\mathrm{th}}$. Furthermore, we can see that, regardless of the transmit power and channel realizations, the SINR for optimal linear receivers is always upper bounded by $\frac{1}{[\boldsymbol{D}]_{k k}-1}$, where $[\boldsymbol{D}]_{k k}-1=\sum_{i \neq l}^{L}\left(\beta_{l i k} / \beta_{l l k}\right)^{2}$ which represents the effect of interference from other cells. This result implies that with OLRs, the pilot contamination effect persists.

\section{Performance AnAlysis for Optimal Linear RECEIVERS}

\section{A. Achievable Rate}

Given (18), the uplink achievable ergodic rate from the $k$ th user in the $l$ th cell to its $\mathrm{BS}$ is given by

$$
\begin{aligned}
R_{\mathrm{opt}, k} & =\mathbb{E}\left\{\log _{2}\left(1+\mathrm{SINR}_{\mathrm{opt}, k}\right)\right\} \\
& =\mathbb{E}\left\{\log _{2}\left(1+\frac{\left[\boldsymbol{D}^{-1}\right]_{k k} X}{\left(1-\left[\boldsymbol{D}^{-1}\right]_{k k}\right) X+1}\right)\right\} \\
& =\mathbb{E}\left\{\log _{2}\left(\frac{1}{1-\left[\boldsymbol{D}^{-1}\right]_{k k}+\left[\boldsymbol{D}^{-1}\right]_{k k} /(X+1)}\right)\right\} .
\end{aligned}
$$

By using the approximate distribution of $X$ given by (20), we derive a closed-form approximation for the uplink achievable ergodic rate as follows.

Proposition 2: The uplink achievable ergodic rate of the $k$ th user in the $l$ th cell for an OLR can be approximated as

$$
\begin{aligned}
& R_{\mathrm{opt}, k} \approx \frac{\log _{2} e}{\Gamma\left(\alpha_{k}\right)} G_{3,2}^{1,3}\left(\begin{array}{l|l}
\theta_{k} & \begin{array}{l}
1-\alpha_{k}, 1,1 \\
1,0
\end{array}
\end{array}\right)
\end{aligned}
$$

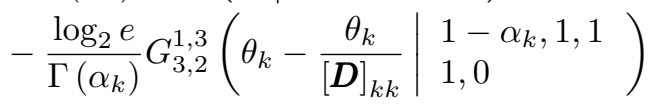

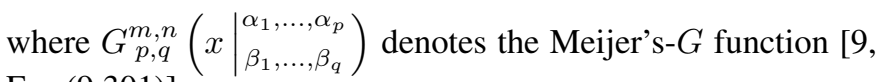
Eq. (9.301)].

Proof: From (29), we have

$$
\begin{aligned}
R_{\mathrm{opt}, k} & =\mathbb{E}\left\{\log _{2}(X+1)\right\}-\mathbb{E}\left\{\log _{2}\left(\left(1-\left[\boldsymbol{D}^{-1}\right]_{k k}\right) X+1\right)\right\} \\
& \approx \int_{0}^{\infty} \log _{2}(x+1) \frac{x^{\alpha_{k}-1} e^{-x / \theta_{k}}}{\Gamma\left(\alpha_{k}\right) \theta_{k}^{\alpha_{k}}} d x \\
& -\int_{0}^{\infty} \log _{2}\left(\left(1-\left[\boldsymbol{D}^{-1}\right]_{k k}\right) x+1\right) \frac{x^{\alpha_{k}-1} e^{-x / \theta_{k}}}{\Gamma\left(\alpha_{k}\right) \theta_{k}^{\alpha_{k}}} d x .
\end{aligned}
$$

Define

$$
\mathcal{I}(a, \alpha, \theta) \triangleq \int_{0}^{\infty} \log _{2}(a x+1) x^{\alpha-1} e^{-\theta x} d x .
$$

To evaluate the integral $\mathcal{I}(a, \alpha, \theta)$, we first use [10, Eq. (8.4.6.5)] to express $\log _{2}(a x+1)$ in terms of a Meijer's $G$-function as follows

$$
\log _{2}(a x+1)=\log _{2} e G_{2,2}^{1,2}\left(\begin{array}{l|l}
a x & 1,1 \\
1,0
\end{array}\right) .
$$

Then, using [10, Eq. (2.24.3.1)], we obtain

$$
\mathcal{I}(a, \alpha, \theta)=\log _{2} e \theta^{-\alpha} G_{3,2}^{1,3}\left(\begin{array}{l|l}
\frac{a}{\theta} & \begin{array}{l}
1-\alpha, 1,1 \\
1,0
\end{array}
\end{array}\right) .
$$

Substituting (34) into (31), we arrive at the desired result (30).

In addition to the result given by Proposition 2, we now propose an analytical lower bound on the achievable ergodic rate which is easier to evaluate. By the convexity of $\log _{2}(1 / x)$ and using Jensen's inequality, we have $R_{\text {opt }, k} \geq \tilde{R}_{\text {opt }, k}$, where

$$
\tilde{R}_{\mathrm{opt}, k} \triangleq-\log _{2}\left(1-\left[\boldsymbol{D}^{-1}\right]_{k k}+\left[\boldsymbol{D}^{-1}\right]_{k k} \mathbb{E}\left\{\frac{1}{1+X}\right\}\right) \text {. }
$$

Proposition 3: The lower bound on the uplink achievable ergodic rate of the $k$ th user in the $l$ th cell for an OLR can be approximated as

$$
\tilde{R}_{\mathrm{opt}, k} \approx-\log _{2}\left(1-\left[\boldsymbol{D}^{-1}\right]_{k k}+\left[\boldsymbol{D}^{-1}\right]_{k k}{ }_{2} F_{0}\left(\alpha_{k}, 1 ;-;-\theta_{k}\right)\right)
$$

where ${ }_{p} F_{q}(\cdot)$ is the generalized hypergeometric function [9, Eq. (9.14.1)], while $\alpha_{k}$ and $\theta_{k}$ are given by (21) and (22), respectively.

Proof: From (20), we have

$$
\begin{aligned}
\mathbb{E}\left\{\frac{1}{X+1}\right\} & \approx \int_{0}^{\infty} \frac{1}{x+1} \frac{x^{\alpha_{k}-1} e^{-x / \theta_{k}}}{\Gamma\left(\alpha_{k}\right) \theta_{k}^{\alpha_{k}}} d x \\
& =\frac{e^{1 / \theta_{k}}}{\theta_{k}^{\alpha_{k}}} \Gamma\left(1-\alpha_{k}, \frac{1}{\theta_{k}}\right)
\end{aligned}
$$

where the last equality is obtained by using [9, Eq. (3.383.10)] and $\Gamma(a, x)=\int_{x}^{\infty} t^{a-1} e^{-t} d t$ being the upper incomplete gamma function [9, Eq. (8.350.2)]. Typically, the value of $\alpha_{k}$ is very large and the value of $\theta_{k}$ is very small. This causes a numerical instability in the evaluation of $\frac{e^{1 / \theta_{k}}}{\theta_{k}^{\alpha_{k}}} \Gamma\left(1-\alpha_{k}, \frac{1}{\theta_{k}}\right)$. 


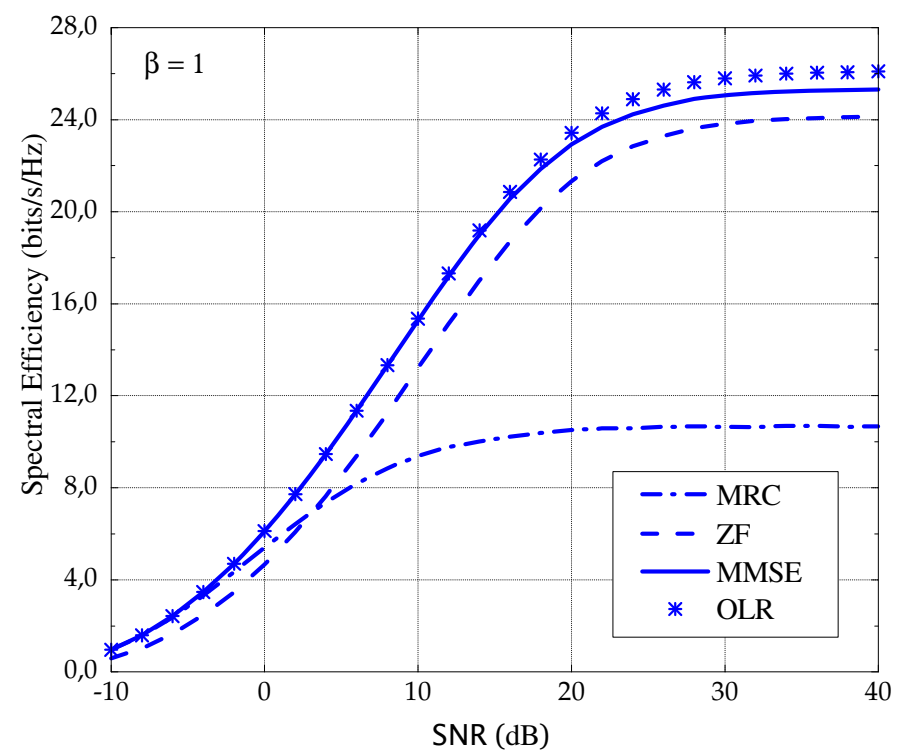

Fig. 1. Spectral efficiency for MRC, ZF, typical MMSE, and optimal linear receivers $(N=20$ and $\beta=1)$.

For this reason, we transform this formula in terms of the generalized hypergeometric function as follows:

$$
\frac{e^{1 / \theta_{k}}}{\theta_{k}^{\alpha_{k}}} \Gamma\left(1-\alpha_{k}, \frac{1}{\theta_{k}}\right)={ }_{2} F_{0}\left(\alpha_{k}, 1 ;-;-\theta_{k}\right)
$$

where (38) stems from [11, Eqs. (07.34.03.0396.01) and (07.31.26.0004.01)]. Substituting (38) into (37), we obtain the desired result in Proposition 3.

\section{B. Large $N$ Analysis}

Without significant loss of generality, we assume $p_{\mathrm{p}}=p_{\mathrm{u}}$. With $p_{\mathrm{u}}=E_{\mathrm{u}} / \sqrt{N}$, from (18), when $N$ grows large, we have

$$
\begin{aligned}
& \operatorname{SINR}_{\mathrm{opt}, k} \stackrel{a . s .}{\rightarrow} \frac{1}{1-\left[\boldsymbol{D}^{-1}\right]_{k k}+\left[\boldsymbol{D}^{-1}\right]_{k k}\left(\tau E_{\mathrm{u}}^{2} \sum_{i=1}^{L} \beta_{l i k}^{2}+1\right)^{-1}}-1 \\
&=\frac{\tau E_{\mathrm{u}}^{2} \beta_{l l k}^{2}}{\tau E_{\mathrm{u}}^{2} \sum_{i=1, i \neq l}^{L} \beta_{l i k}^{2}+1}
\end{aligned}
$$

where $\stackrel{\text { a.s. }}{\rightarrow}$ denotes almost sure convergence, and the limit is obtained by using the law of large numbers. We can see that with OLR and very large antenna arrays at the BS, we can reduce the transmit power proportionally to $1 / \sqrt{N}$

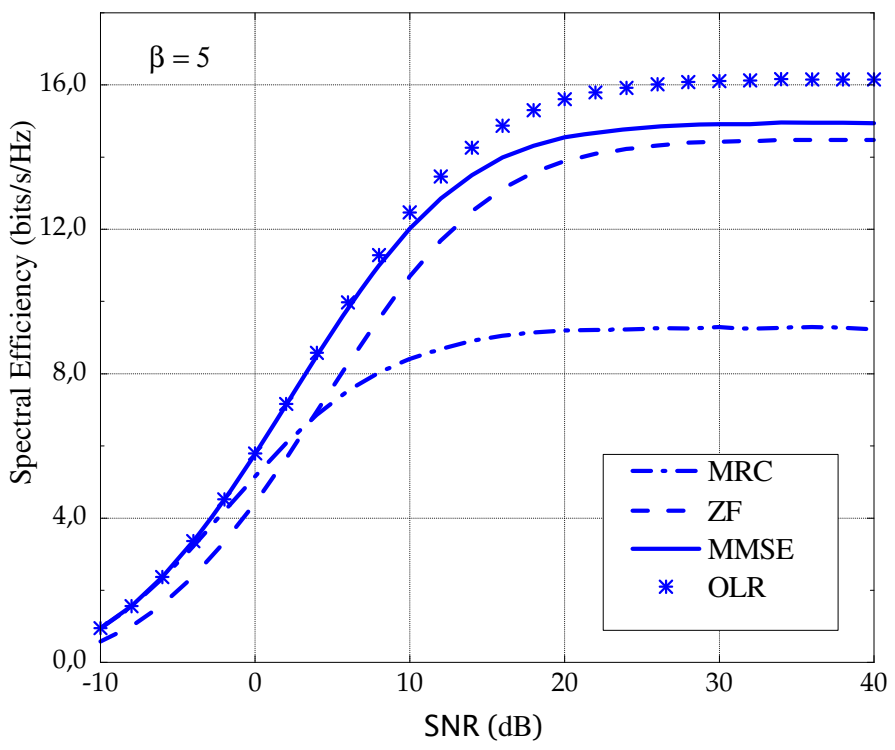

Fig. 2. Spectral efficiency for MRC, ZF, typical MMSE, and optimal linear receivers $(N=20$ and $\beta=5)$.

while maintaining a desired quality-of-service. Furthermore, we can see that the above result coincides with the asymptotic results of MRC, ZF, and MMSE in [4]. This means that when the number of BS antennas is large, MRC, ZF, and MMSE perform almost as well as OLR.

\section{NumericAl RESUlts}

Consider a cellular network with $L=7$ cells. Each cell serves 10 users $(K=10)$. The large-scale fading matrix $\boldsymbol{D}_{1 i}, i=1, \ldots, 7$, is chosen as shown at the bottom of the page, where $\beta$ is the intercell interference factor which represents the effect of the interference from other cells. ${ }^{2}$ Unless otherwise stated, we choose $\beta=1$. Furthermore, we choose $T=196$, $\tau=10, p_{\mathrm{p}}=p_{\mathrm{u}}$, and define $\mathrm{SNR} \triangleq p_{\mathrm{u}}$. We consider the spectral efficiency (sum-rate in bits/s/Hz) in cell 1 which is

${ }^{2}$ To examine the performance in a practical scenario, the large-scale fading coefficients are chosen as follows: we consider hexagonal cells which have a radius of 1000 meters. We assume that the users are located randomly and uniformly in the cell and the distance between the user and the base station is greater than 100 meters. The large-scale fading is modelled via a log-normal distribution with a standard deviation of $8 \mathrm{~dB}$ and the path loss exponent is equal to 4 . By simulation, one realization of large-scale fading is chosen.

$$
\begin{aligned}
& \boldsymbol{D}_{11}=10^{-3} \operatorname{diag}[29.0281,0.3199,908.2751,286.4841,0.5250,3.8211,0.7230,11.2149,3.3877,87.2929] \\
& \boldsymbol{D}_{12}=10^{-3} \beta \times \operatorname{diag}[0.0135,0.1112,0.0210,0.5061,1.2007,0.2386,0.0461,0.0109,0.6734,0.0083] \\
& \boldsymbol{D}_{13}=10^{-3} \beta \times \operatorname{diag}[1.6199,0.0028,0.4856,0.1218,0.0108,0.0046,0.0319,0.0239,0.0001,0.0475] \\
& \boldsymbol{D}_{14}=10^{-3} \beta \times \operatorname{diag}[0.0684,0.0130,0.0236,0.0692,0.2540,0.1977,0.0068,0.0037,0.3747,1.0689] \\
& \boldsymbol{D}_{15}=10^{-3} \beta \times \operatorname{diag}[2.5533,0.0189,0.9230,0.0112,0.0089,0.6919,0.0391,0.3025,0.1817,0.2307] \\
& \boldsymbol{D}_{16}=10^{-3} \beta \times \operatorname{diag}[0.1040,0.1345,0.0234,1.3590,0.3573,0.0022,0.0562,0.0173,0.0719,0.3601] \\
& \boldsymbol{D}_{17}=10^{-3} \beta \times \operatorname{diag}[1.5309,0.3749,0.3129,0.0647,0.0210,0.0775,0.1489,0.1797,12.8758,0.0660] .
\end{aligned}
$$


defined as

$$
\mathcal{S}=\left(1-\frac{\tau}{T}\right) \sum_{k=1}^{K} R_{k}
$$

where $T$ is the coherence time interval (in symbols) and $R_{k}$ is the achievable uplink ergodic rate of the $k$ th user in cell 1 .

Figures 1 and 2 show the spectral efficiency for OLR, typical MMSE, ZF, and MRC, with different intercell interference factors $\beta=1$ and $\beta=5$, respectively. Here we choose $N=20$. We can see that at low SNR (and hence, low spectral efficiency), MRC performs as well as OLR, and better than ZF. However, at high SNR, ZF performs better than MRC. OLR always performs the best across the SNR range. In particular, we can see that when the effect of intercell interference increases, the performance gap between OLR and typical MMSE increases, thereby revealing the optimality of the proposed receiver. This is due to the fact that the OLR takes the correlation between intercell interference and the channel estimate into account.

To validate the tightness of our analytical approximation, we consider the approximately bounded and the exact spectralefficiencies of OLR as Fig. 3. The "Analytical Approximation" curves are obtained by using Proposition 3, and the "Simulation" curves are generated from the outputs of a MonteCarlo simulation using (18). We can see that our bound is very tight, especially at large $N$. At high SNR, we cannot improve the system performance by using more power. The reason is that when we increase the transmit power then the interference from other cells also increases, and hence the system is interference-limited. To improve the system performance, instead of increasing the transmit power, we can deploy more antennas at the BS. We can see that when $N$ increases the spectral efficiency increases significantly.

\section{CONCLUSION}

In this paper, we proposed a novel OLR for multicell MUMIMO systems where the channel is estimated using uplink training. Our proposed OLR takes the correlation between the channel estimate and the interference into account. Therefore, the OLR is always better than MRC, ZF, or typical MMSE receivers, and its performance is very close to the latter. We derived the statistical distribution of the received SINR for this OLR. We then deduced an analytical approximation of the exact and lower bound on the achievable ergodic rate. The latter approximation is very tight, especially at large number of BS antennas. Furthermore, as for MRC, ZF, and MMSE, we showed that for OLR, large antenna arrays enable us to cut the transmit power of each user proportionally to $1 / \sqrt{N}$.

\section{ACKNOWLEDGMENT}

The work of H. Q. Ngo and E. G. Larsson was supported in part by the Swedish Research Council (VR), the Swedish Foundation for Strategic Research (SSF), and ELLIIT. The work of M. Matthaiou was supported in part by the Swedish Governmental Agency for Innovation Systems (VINNOVA) within the VINN Excellence Center Chase.

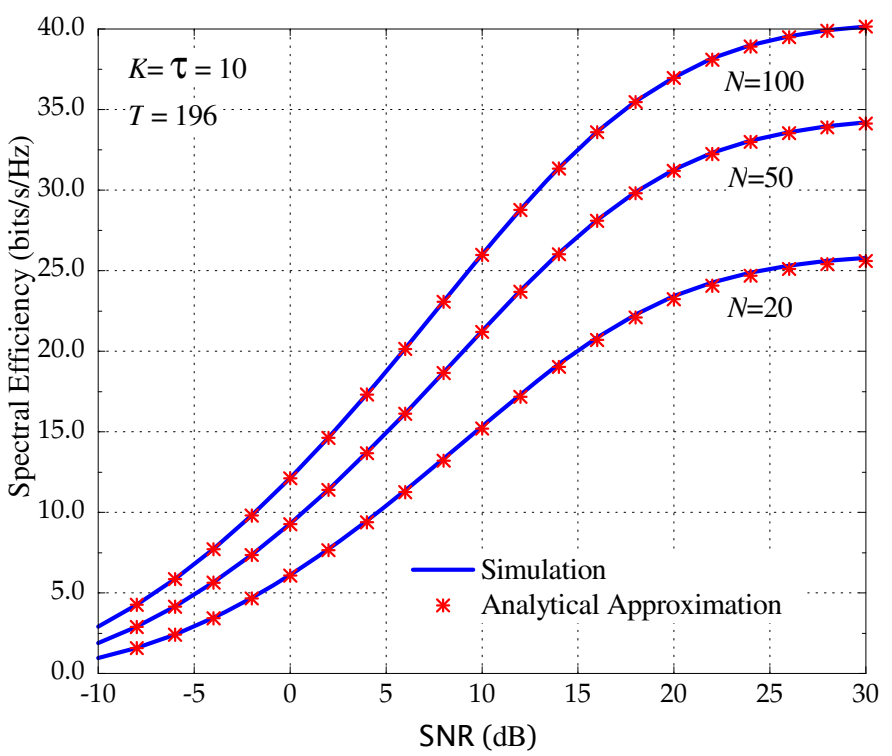

Fig. 3. Spectral-efficiency for the optimal linear receiver $(L=7, K=\tau=$ 10 , and $T=196$ ).

\section{REFERENCES}

[1] T. L. Marzetta, "Noncooperative cellular wireless with unlimited numbers of base station antennas," IEEE Trans. Wireless Commun., vol. 9, no. 11, pp. 3590-3600, Nov. 2010.

[2] F. Rusek, D. Persson, B. K. Lau, E. G. Larsson, T. L. Marzetta, O. Edfors, and F. Tufvesson, "Scaling up MIMO: Opportunities and challenges with very large arrays," IEEE Signal Process. Mag., to appear. [Online]. Available: http://arxiv.org/abs/1201.3210.

[3] J. Jose, A. Ashikhmin, T. L. Marzetta, and S. Vishwanath, "Pilot contamination and precoding in multi-cell TDD systems," IEEE Trans. Wireless Commun., vol. 10, no. 8, pp. 2640-2651, Aug. 2011.

[4] H. Q. Ngo, E. G. Larsson, and T. L. Marzetta, "Energy and spectral efficiency of very large multiuser MIMO systems," IEEE Trans. Commun., submitted. [Online]. Available: http://arxiv.org/abs/1112.3810.

[5] J. Hoydis, S. ten Brink, and M. Debbah, "Massive MIMO in the UL/DL of cellular networks: How many antennas do we need?" IEEE J. Sel. Areas Commun., 2012, accepted.

[6] S. M. Kay, Fundamentals of Statistical Signal Processing: Estimation Theory, Englewood Cliffs, NJ: Prentice Hall, 1993.

[7] N. Kim, Y. Lee, and H. Park, "Performance analysis of MIMO system with linear MMSE receivers," IEEE Trans. Wireless Commun., vol. 7, no. 11, pp. 4474-4478, Nov. 2008.

[8] L. Hong and A. G. Armada, "Bit error rate performance of MIMO MMSE receivers in correlated Rayleigh flat-fading channels," IEEE Trans. Veh. Technol., vol. 60, no. 1, pp. 313-317, Jan. 2011.

[9] I. S. Gradshteyn and I. M. Ryzhik, Table of Integrals, Series, and Products, 7th ed. San Diego, CA: Academic, 2007.

[10] A. P. Prudnikov, Y. A. Brychkov, and O. I. Marichev, Integrals and Series. New York: Gordon and Breach Science, 1990, vol. 3.

[11] Wolfram, "The Wolfram functions site." [Online]. Available: http://functions.wolfram.com 